\title{
An empirical behavioral order-driven model with price limit rules
}

\author{
Gao-Feng Gu $u^{1,2}$, Xiong Xiong ${ }^{3,4}$, Hai-Chuan X ${ }^{1,2}$, Wei Zhang ${ }^{3,4}$, Yongjie Zhang ${ }^{3,4}$, Wei Chen ${ }^{5}$ and \\ Wei-Xing Zhou ${ }^{1,2,6^{*}}$ (D)
}

${ }^{*}$ Correspondence: wxzhou@ecust.edu.cn

${ }^{1}$ School of Business, East

China University of Science and Technology, Shanghai,

China

Full list of author information is available at the end of the article

\begin{abstract}
We propose an empirical behavioral order-driven (EBOD) model with price limit rules, which consists of an order placement process and an order cancellation process. All the ingredients of the model are determined based on the empirical microscopic regularities in the order flows of stocks traded on the Shenzhen Stock Exchange. The model can reproduce the main stylized facts in real markets. Computational experiments unveil that asymmetric setting of price limits will cause the stock price to diverge exponentially when the up price limit is higher than the down price limit and to vanish vice versa. We also find that asymmetric price limits have little influence on the correlation structure of the return series and the volatility series, but cause remarkable changes in the average returns and the tail exponents of returns. Our EBOD model provides a suitable computational experiment platform for academics, market participants, and policy makers.
\end{abstract}

Keywords: Econophysics, Order-driven model, Agent-based model, Asymmetric price limit, Stylized facts, Limit order book

\section{Introduction}

The emerging Chinese stock market adopted the price limit mechanism on 2 January 1997 to refrain from speculative behaviors and stabilize the market. The mechanism sets symmetric price limits in which the up limit $\phi_{+}=10 \%$ and the down limit $\phi_{-}=-10 \%$ for common stocks and $\phi_{+}=5 \%$ and $\phi_{-}=-5 \%$ for specially treated (ST and "ST) stocks, ${ }^{1}$ also called stocks bearing risk warnings. On 27 July 2012 (Friday), after the closing of the Chinese stock market, the Shanghai Stock Exchange (SSE) released the Draft Guideline on the Trading of Stocks Bearing Risk Warnings. A key term (Article VII) suggested that, for stocks bearing risk warnings, the maximum percentage of price increase is $\phi_{+}=1 \%$, while the maximum percentage of price decrease is $\phi_{-}=-5 \%$. Through the Draft, especially Article VII, SSE wanted to curb the vicious speculation on junk stocks in the market, guide funds to blue-chip stocks and promote the development of the stock market. Impacted by this event, on 30 July 2012, 106 out of the 110 ST and "ST shares were sealed at the down price limit. On that day, the SHSZ Composite Index, the

\footnotetext{
${ }^{1}$ An ST stock means its net profit of the last two years is negative, which is used to warn investors of investment risks. A
} "ST stock means its net profit of the last three years is negative, which is used to warn investors of delisting risks.

(c) The Author(s), 2021. Open Access This article is licensed under a Creative Commons Attribution 4.0 International License, which permits use, sharing, adaptation, distribution and reproduction in any medium or format, as long as you give appropriate credit to the original author(s) and the source, provide a link to the Creative Commons licence, and indicate if changes were made. The images or other third party material in this article are included in the article's Creative Commons licence, unless indicated otherwise in a credit line to the material. If material is not included in the article's Creative Commons licence and your intended use is not permitted by statutory regulation or exceeds the permitted use, you will need to obtain permission directly from the copyright holder. To view a copy of this licence, visit http:// creativecommons.org/licenses/by/4.0/. 
Shenzhen Stock Exchange (SZSE) Composite Index, and the Chinese Stock Market 500 Index (CSI 500) dropped respectively $-0.89 \%,-1.65 \%$ and $-2.01 \%$.

This Draft, especially Article VII, incurred a lot of opposition. Yu Chen, the general manager of Beijing Shennong Capital Management, released an open letter to the SSE, objecting to the asymmetric price limit setting on risk-warning stocks. He argued that the Exchange should not enable unfair trading rules since the provision of asymmetric price limits would significantly increase the cost of buying and holding risk-warning stocks. He posited that under the asymmetric price limits, the prices of ST and "ST stocks would be more likely to fall. He urged that Article VII should be removed from the Draft. The final Guideline did replace the asymmetric price limits with symmetric limits. However, is there any technical evidence supporting these "arguments"? In this work, we design an empirical behavioral order-driven model. Armed with this new model, computational experiments show that the asymmetric setting with a larger down limit $\left(\phi_{+}<\left|\phi_{-}\right|\right)$leads to vanishing prices. Hence, under the Draft Guideline, no stock can escape the fate of being delisted once it is labeled risk warning.

Price limits and trading halts are important mechanisms in many markets (Westerhoff 2003; Ehrenstein and Westerhoff 2006; Westerhoff 2006). Some studies design agentbased models and perform computational experiments to investigate the effects of price limits. Yeh and Yang find no evidence of volatility spillover but phenomena of delayed price discovery and trading interference, whose significance depends on the level of the price limits (Yeh and Yang 2013). Zhang et al. demonstrate that both price limits can cause a volatility spillover effect and a trading interference effect (Zhang et al. 2016). Moreover, some researchers argue that a proper level of price limits is helpful to stabilize the markets (Yeh and Yang 2010; Xiong et al. 2015). Inspired by the asymmetric effect of symmetric price limit mechanism in the Chinese stock market, Li and Geng perform Monte Carlo simulations of the VF-EGARCH-M model and conclude that there is an optimal design of asymmetric price limit mechanism with $\phi_{+}>\left|\phi_{-}\right|(\mathrm{Li}$ and Geng 2012). However, our computational experiments show that asymmetric price limit rules do hurt the market.

The Chinese stock market is an order-driven market that adopts the continuous double auction mechanism and symmetric price limits mechanism (Gu et al. 2007; Jiang et al. 2008; Mu et al. 2009; Wu et al. 2017). Price limits play an important role in price formation (Telser 1981; Brennan 1986; Subrahmanyam 1994; Kim and Rhee 1997; Goettler et al. 2009; Hsieh et al. 2009; Wong et al. 2009; Wan et al. 2015, 2018). The model proposed in this work was inspired by the seminal Mike-Farmer model (Mike and Farmer 2008) and its updated version in Gu and Zhou's model (2009a). This family of order-driven models mimics the order placement process and order cancellation process entering the trading terminal. The order placement process contains three components: order direction, relative order price, and order size. The microscopic rules of these models are obtained by empirical regularities of these processes. Compared with existing models (Mike and Farmer 2008; Gu and Zhou 2009a), the new model introduced in this work makes two innovations. First, it adopts a new definition of the relative order price by integrating price limits $\phi_{+}$and $\phi_{-}$as model parameters. This enables us to investigate the mechanisms of phenomena caused by price limits that we will briefly review below. Second, it considers order sizes. Hence, the model is more realistic and significantly 
enhances its ability for the study of market microstructure theories such as immediate price impacts (Lillo et al. 2003; Lim and Coggins 2005; Zhou 2012a, b; Xu et al. 2017; Pham et al. 2017). The models in Mike and Farmer (2008), Gu and Zhou (2009a) have been applied to understand the mechanisms underlying stylized facts (Gu and Zhou 2009b; Meng et al. 2012; Zhou et al. 2017).

The study of order-driven models has a long history, which may have begun more than 50 years ago (Stigler 1964). Researchers have constructed different order-driven models based on different groups of micro-driving rules, attempting to simulate the dynamics of the limit-order book (Maslov 2000; Farmer et al. 2005; Mike and Farmer 2008; Gu and Zhou 2009a; Tseng et al. 2010). In these models, orders are not specific to certain traders and the traders have zero intelligence. Other behavioral models include percolation models (Stauffer 1998; Cont and Bouchaud 2000; Eguíluz and Zimmermann 2000), Ising models (Föellmer 1974; Chowdhury and Stauffer 1999; Iori 1999; Kaizoji 2000; Bornholdt 2001; Zhou and Sornette 2007), minority games (Arthur 1994; Challet and Zhang 1997; Challet et al. 2000, 2001a, b, 2005), and so on. Another important family is heterogeneous agent models, in which the agents are combinations of informed traders, fundamentalists, technical traders, smart traders, noise traders, and so on (Brock and Hommes 1997, 1998; Lux and Marchesi 1999; Chiarella and Iori 2002; Chiarella et al. 2006; Barunik et al. 2009; Xu et al. 2014a, b). Under this framework, one can study the effects of diverse factors, such as noise (Chiarella et al. 2011), technical trading rules (Chiarella et al. 2009; He and Li 2015), and investor sentiment (Chiarella et al. 2017). Excellent reviews have been provided in Chakraborti et al. (2011), Sornette (2014), Zha et al. (2020). Nevertheless, the effects of asymmetric price limits have not been studied with computational experiments within these models. We note that insightful detailed discussions on how to run policy experiments with agent-based stock market models can be found in Westerhoff (2008), Westerhoff and Franke (2018).

The rest of the paper is organized as follows. Section 2 describes briefly the database we adopt and present the stylized facts relevant to the construction of the model. Section 3 constructs and validates the behavioral order-driven model based on empirical regularities of order flows. We perform computational experiments in Sect. 4 to study the effects of asymmetric price limit rules on the evolution of stock price and several stylized facts. Section 5 summarizes the results.

\section{Relevant stylized facts}

\section{Data sets}

We use the ultra-high-frequency order flow data of $32 \mathrm{~A}$-share stocks and $11 \mathrm{~B}$-share stocks traded on the Shenzhen Stock Exchange (SZSE) in 2003 to build the order-driven model. The 32 A-shares were constituents of the Shenzhen Stock Exchange Component Index and the $11 \mathrm{~B}$-share stocks paired with $11 \mathrm{~A}$-share stocks in our sample.

Each entry of the records contains the details of order placement and order cancellation, including the order placement/cancellation time, order price, order size, and order identifier which identifies whether the order is a buy order, a sell order, or a cancellation. The timestamp of the database is accurate to $0.01 \mathrm{~s}$. The data allows us to reconstruct the limit order books (LOBs) and reproduce the price formation process. 

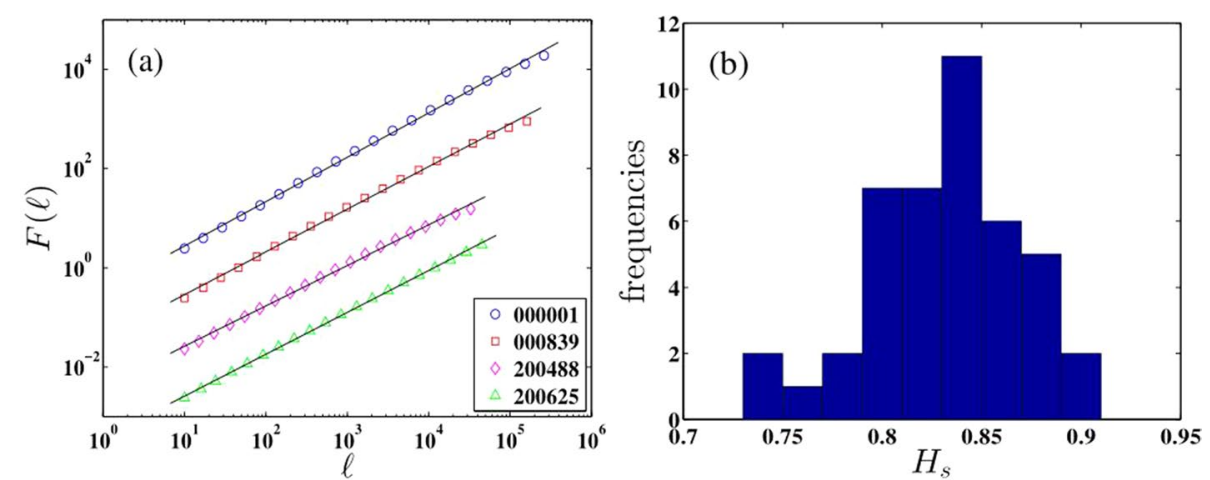

Fig. 1 Long memory in order directions. a Plots of the fluctuation functions $F(\ell)$ of order directions for four stocks $000001,000839,200488$ and 200625 . The solid lines are the least squares fits to the data. The scaling curves for 000839,200488 and 200625 have been shifted vertically for clarity. b Histograms of Hurst exponents $\mathrm{H}_{s}$ of order directions for all the 43 stocks

\section{Order direction}

The first ingredient of order placement is the order direction $s$. Assuming $s=+1$ for buy orders and $s=-1$ for sell orders, we can construct the order direction series for each stock. To measure the memory effect of the order direction series for all the 43 stocks, we apply the Detrending Moving Average Analysis (DMA) (Carbone 2009; Gu and Zhou 2010; Jiang et al. 2019), which is a popular method for estimating the Hurst exponent (Jiang and Zhou 2011; Shao et al. 2012). The detrending fluctuation function $F(\ell)$ can be computed and is expected to be power-law related to the scale size $\ell$, which reads

$$
F(\ell) \sim \ell^{H},
$$

where $H$ is the DMA scaling exponent or roughly the Hurst exponent. The time series is persistent if $H>0.5$, uncorrelated if $H=0.5$, and antipersistent if $H<0.5$. A persistent time series has a long memory. We use $H_{s}$ with subscript $s$ for the Hurst exponents of order directions.

The fluctuation functions $F(\ell)$ with respect to the scale $\ell$ of four randomly chosen stocks (000001 and 000839 are A shares and 200488 and 200625 are B shares) are illustrated in Fig. 1a. Excellent power-law scaling relations are observed in the scaling ranges over 4 to 5 orders of magnitude. The Hurst exponents of the four stocks are estimated to be $H_{s}=0.895 \pm 0.008$ for stock 000001, $H_{s}=0.859 \pm 0.009$ for stock 000839, $H_{s}=0.814 \pm 0.009$ for stock 200488, $H_{s}=0.845 \pm 0.005$ for stock 200625. The Hurst exponents of all the 43 stocks are presented in Table 1. We also show the histogram of Hurst exponents of all the stocks in Fig. 1b. It is clear that all the Hurst exponents are all larger than 0.5 and we document that the order direction series significantly has a long memory. According to Table 1, the mean Hurst exponent for all the stocks is $\left\langle H_{s}\right\rangle=0.841 \pm 0.036$. Moreover, the mean Hurst exponent of the A shares is $\left\langle H_{s, A}\right\rangle=0.854 \pm 0.027$, which is a little larger than that of $\mathrm{B}$ shares $\left\langle H_{s, B}\right\rangle=0.806 \pm 0.036$. It indicates that stronger persistence exists in the A shares in 2003 in the Chinese stock markets. The results are consistent with the findings of Lillo and Farmer for the London Stock Exchange (LSE) stocks (Lillo and Farmer 2004). However, we find that the SZSE stocks have stronger persistence in order directions than the 
LSE stocks, which reflects the fact that there are stronger imitative and herding behaviors in the Chinese stock market.

\section{Relative price}

We define the relative price $x$ based on the price limit rules in the Chinese stock market, which is different from the definition of Mike and Farmer for LSE stocks (Mike and Farmer 2008). We require that the relative price $x$ varies in the range $[-1,1]$ and stands for the order aggressiveness ${ }^{2}$. When a buy order is placed on the down limit or a sell order is placed on the up limit, we have $x=-1$. These orders are the least aggressive. When a buy order is placed on the up limit or a sell order is placed on the down limit, we have $x=1$. These orders are the most aggressive. When an order is placed on the opposite best price, i.e., $p_{t}=p_{a}(t-1)$ for buy orders and $p_{t}=p_{b}(t-1)$ for sell orders, we have $x=0$. Following these considerations, we define the relative price as follows:

$$
x_{t}= \begin{cases}{\left[p_{b}(t-1)-p_{t}\right] /\left[p_{b}(t-1)-p_{\min }(T)\right]} & \text { for sell market orders } \\ {\left[p_{b}(t-1)-p_{t}\right] /\left[p_{\max }(T)-p_{b}(t-1)\right]} & \text { for sell limit orders } \\ {\left[p_{t}-p_{a}(t-1)\right] /\left[p_{\max }(T)-p_{a}(t-1)\right]} & \text { for buy market orders } \\ {\left[p_{t}-p_{a}(t-1)\right] /\left[p_{a}(t-1)-p_{\min }(T)\right]} & \text { for buy limit orders }\end{cases}
$$

where $p_{t}$ is the price of order placed at event time $t, p_{a}(t-1)$ and $p_{b}(t-1)$ are the best ask and best bid at event time $t-1$, and $p_{\max }(T)$ and $p_{\min }(T)$ are the maximum and minimum valid prices on a trading day $T$. Larger $x$ values imply that traders are more eager to make a transaction immediately and the orders are thus more aggressive. Effective market orders $(x \geq 0)$ result in an immediate transaction, while effective limit orders $(x<0)$ are stored in the limit order book waiting to be executed by future effective market orders on the opposite side. Therefore, the aggressiveness decreases from positive to zero, then to negative when the order is placed in the opposite limit order book, at the opposite best price, in the bid-ask spread, at the same best price and in the same limit order book.

If the price is at the up limit when an order arrives at $t$, we have

$$
p_{a}(t-1)=p_{b}(t-1)=p_{\max }(T) .
$$

Under such scenarios, the relative prices of sell market orders and buy limit orders are well defined by Eq. (2)

$$
x_{t}=\left\{\begin{array}{ll}
{\left[p_{b}(t-1)-p_{t}\right] /\left[p_{b}(t-1)-p_{\min }(T)\right]} & \text { for sell market orders } \\
{\left[p_{t}-p_{a}(t-1)\right] /\left[p_{a}(t-1)-p_{\min }(T)\right]} & \text { for buy limit orders }
\end{array},\right.
$$

while the relative prices of buy market orders and sell limit orders cannot be defined by Eq. (2). For buy market orders, one requires that $p_{t}>p_{b}(t)=p_{\max }(T)$, which is however not permitted. For sell limit orders, one requires $p_{t}>p_{a}(t)=p_{\max }(T)$, which is again not permitted. In other words, when the price is at the up limit, both buy market orders and sell limit orders do not exist. Hence, we simply pose that

$$
p_{t}=p_{\max }(T)
$$

\footnotetext{
${ }^{2}$ Note that aggressive orders are orders that move prices (Degryse et al. 2005). Here we extend the concept of "aggressiveness".
} 
no matter what the value of $x>0$ is. The situation for down price limit is similar. If the price is at the down limit when an order arrives at $t$, we have

$$
p_{a}(t-1)=p_{b}(t-1)=p_{\min }(T) .
$$

Under such scenarios, the relative prices of buy market orders and sell limit orders are well defined by Eq. (2)

$$
x_{t}=\left\{\begin{array}{ll}
{\left[p_{b}(t-1)-p_{t}\right] /\left[p_{\max }(T)-p_{b}(t-1)\right]} & \text { for sell limit orders } \\
{\left[p_{t}-p_{a}(t-1)\right] /\left[p_{\max }(T)-p_{a}(t-1)\right]} & \text { for buy market orders }
\end{array},\right.
$$

while the relative prices of sell market orders and buy limit orders cannot be defined by Eq. (2). For sell market orders, one requires that $p_{t}<p_{a}(t)=p_{\min }(T)$, which is however not permitted. For buy limit orders, one requires $p_{t}<p_{a}(t)=p_{\min }(T)$, which is also not permitted. In other words, when the price is at the down limit, both sell market orders and buy limit orders do not exist. Therefore, we simply pose that

$$
p_{t}=p_{\min }(T),
$$

regardless of what the value of $x<0$ is.

Figure 2a presents the empirical probability density functions (PDFs) $f(x)$ of relative prices aggregating both buy and sell orders for four representative stocks in the sample. We find that the PDF curves of four stocks almost collapse together, especially in the range $x<0$. Moreover, other stocks have similar probability distributions as presented in Fig. 2b, except for the stock 000720 which has obviously higher probabilities in the range $0<x<0.5$. The $f(x)$ functions reach their maximums around the point $x=0$, which means that many traders tend to place orders at the opposite best price to balance the relationship between the transaction cost and transaction opportunity. The distributions are asymmetric (the skewness equal to -2.69 for stock 000001), which implies that more orders are placed in the limit order book. According to the order flow data of stock 000001 , only $28.28 \%$ of the placed orders are effective market orders with $x \geq 0$. This observation verifies that it is natural to maintain nonempty LOBs. We also find that the values at $x= \pm 1$ are significant jumps, which shows that quite a few traders place extreme orders at the down or up limit. Figure $2 \mathrm{c}, \mathrm{d}$ show the empirical probability density functions $f(x)$ of relative prices without price limits defined in Mike and Farmer (2008) for comparison. Under this definition, the relative price $x$ varies in $[-0.2,0.2]$. The most remarkable feature is that there are "humps" at $x= \pm 10 \%$, which reflects the effect of price limits. In addition, the jumps at $x= \pm 20 \%$ are less significant.

The memory effect of relative prices also plays an important role in model construction by introducing long memory in the volatility (Gu and Zhou 2009a; Zhou et al. 2017). We study the memory effect using the DMA method and Fig. 3a presents the fluctuation function $F(\ell)$ with respect to the scale size $\ell$ of relative prices for four representative stocks. Each curve reveals excellent power-law scaling behavior with the scaling range spanning 4-5 orders of magnitude. Using the least-squares regression method, we obtain the Hurst exponents $H_{x}=0.847 \pm 0.009$ for stock 000001, $H_{x}=0.872 \pm 0.004$ for stock 000839, $H_{x}=0.736 \pm 0.005$ for stock 200488, and $H_{x}=0.763 \pm 0.006$ for stock 200625. The Hurst exponents of relative prices for all the 43 stocks are presented in Table 2 . The values are all significantly greater than 0.5 . We conclude that the relative 

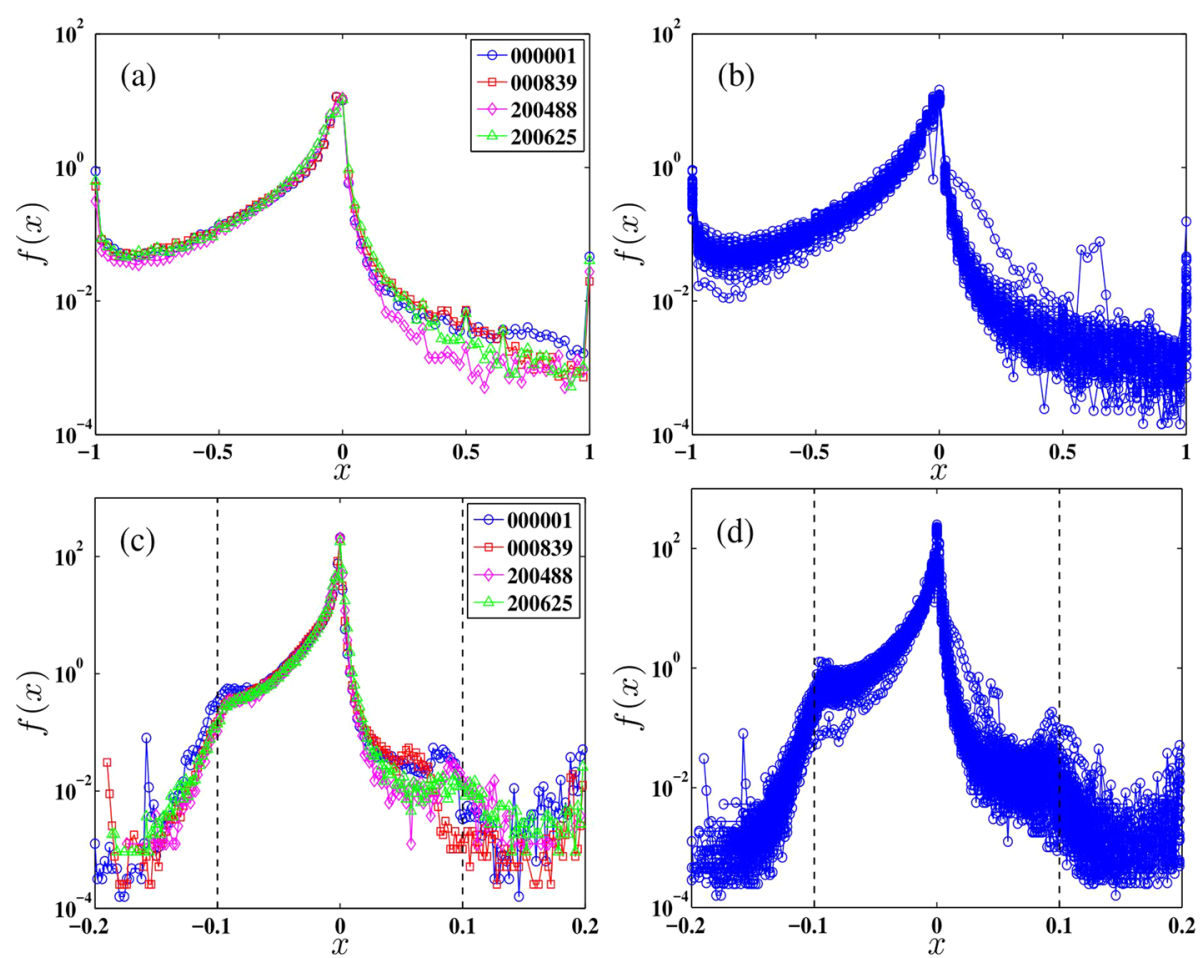

Fig. 2 Empirical probability density function $f(x)$ of relative prices. a, c Two A-share stocks 000001 and 000839 and two B-share stocks 200488 and 200625. b, d All the 43 stocks. The relative prices are defined according to price limits in plots (a) and (b) and according to Mike and Farmer (2008) in plots (c) and (d)
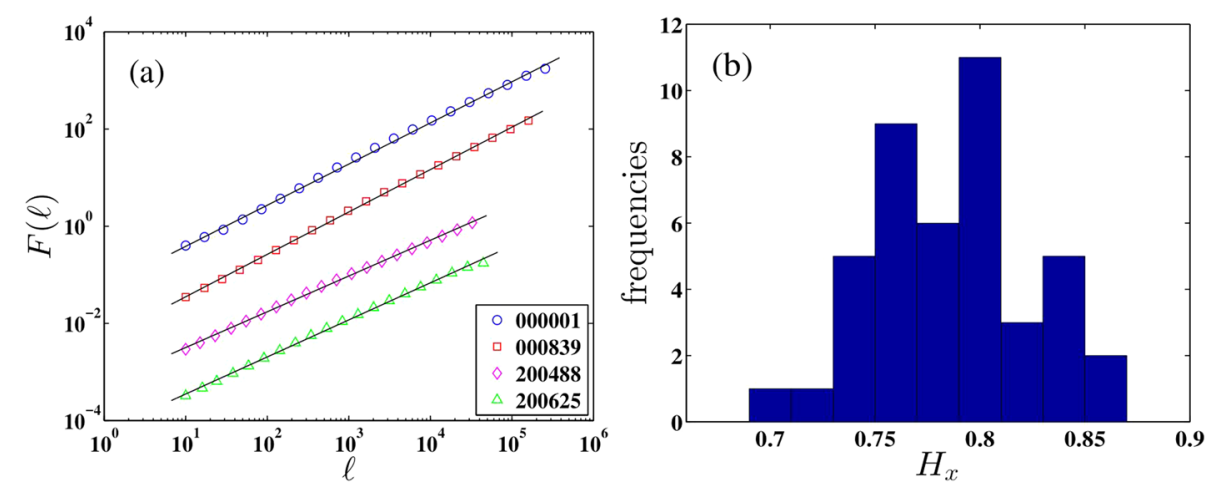

Fig. 3 Long memory in relative order prices. a Plots of the fluctuation functions $F(\ell)$ of relative order prices for four stocks $000001,000839,200488$ and 200625. The solid lines are the least squares fits to the data. The scaling curves for 000839, 200488 and 200625 have been shifted vertically for clarity. b Histograms of Hurst exponents $H_{x}$ of relative order prices for all the 43 stocks

prices of placed orders have a long memory. The histogram of the Hurst exponents of all stocks is showed in Fig. 3b, which confirms the long memory of relative prices in all stocks. According to Table 2, the average Hurst exponents are $\left\langle H_{x}\right\rangle=0.796 \pm 0.035$ for all stocks, $\left\langle H_{x, A}\right\rangle=0.808 \pm 0.030$ for A shares and $\left\langle H_{x, B}\right\rangle=0.761 \pm 0.020$ for B shares. It means that stronger persistence exists in relative prices of A shares in the year 2003. We 

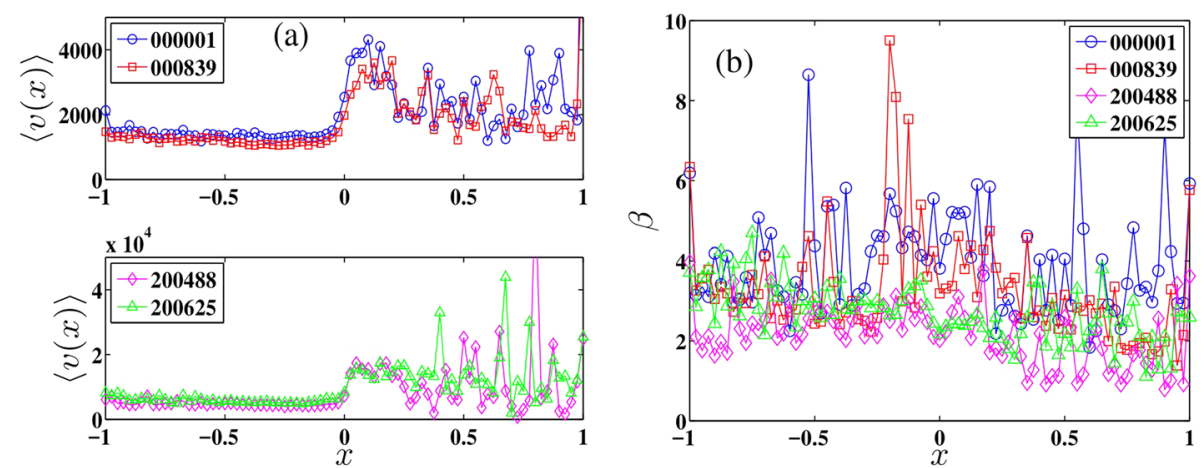

Fig. 4 Dependence of order size with respect to relative price. $\mathbf{a}$ The mean sizes $\langle v(x)\rangle$ against the relative prices $x$ of empirical data for four stocks $000001,000839,200488$ and 200625 . b The ratio $\beta(x)$ as a function of $x$ for the same four stocks

conjecture that the diagonal effect originally unveiled by Biais et al. (1995) is stronger for A-share stocks than for B-share stocks.

We propose a simple linear model between $H_{x}$ and $H_{s}$ and estimate the coefficients. It follows that

$$
H_{x}=0.471+0.466 H_{s}
$$

in which both coefficients are significantly different from 0 at the $3 \%$ significance level and the adjusted R-square is 0.179 . Therefore, we can draw a consistent conclusion that stronger imitative and herding behavior in a stock incurs stronger persistence in the order directions and relative prices.

\section{Order size}

The last ingredient is the order size $v$. In the model, we do not simply study the statistical properties of order size, but analyze the relationship between the order size $v$ and relative price $x$. It is because there is a remarkable number preference in the distribution of order size (Mu et al. 2009), which suggests that the distribution is singular almost everywhere and cannot be generated feasibly.

The relationship between order size and the relative price of the same four representative stocks is presented in Fig. 4a. For each stock, we divided the submitted orders into many groups by binning their relative prices, and the average relative price and average order size are calculated for each group. We find that these curves have similar shapes. Overall, market orders have larger sizes than limit orders. We observe that the average order size $\langle v(x)\rangle$ is almost independent of the relative price for effective limit orders $(x<0)$. There is an interesting feature showing that around the point $x=0$, the average size $\langle v(x)\rangle$ increases rapidly with the relative price $x$, which indicates that traders tend to place larger orders around the opposite price level. For market orders with large $x$ values, the average order size fluctuates a lot. It is because that the number of such market orders is relatively small. Impatient traders submit effective market orders with large relative prices to ensuring execution. However, most orders do not penetrate many levels on the opposite book. Moreover, we find that the order sizes of A-share stocks (000001 and 000839) are smaller than the ones of 
B-share stocks (200488 and 200625). This result is also confirmed by other A shares and B shares stocks, indicating that investors submitted larger orders in the B-share markets, which is because the proportion of retailer traders is much higher in the A-share market.

We assume that the distribution of $v(x)$ is normal for a given $x$. We calculate the ratio $\beta$ which is the standard deviation of order sizes $\sigma(v)$ divided by the average size $\langle v(x)\rangle$

$$
\beta=\sigma(v) /\langle v(x)\rangle .
$$

Figure $4 \mathrm{~b}$ illustrates the relation between the ratio $\beta$ and the relative price $x$ for the four stocks. We find that the ratio $\beta$ almost fluctuates around a constant value for the four stocks. The normality assumption of $v(x)$ will be used to generate the order size $v(x)$ for an order with a relative price $x$.

\section{Order cancellation}

Order cancellation is another main process of continuous double auction. It refers to removing stale orders from the LOB. Order cancellation plays an important role in the price formation of security markets. If all the orders placed at the best price are canceled, the mid-price defined as the mean value of the best bid and best ask will change. If a cancellation takes place inside the LOB, it has a potential impact on price movement. The Mike-Farmer model (2008) considers three factors in the conditional order cancellation probability, that is, the relative distance of the target order to the opposite best, the ratio of buy or sell orders on the book, and the total number of LOB orders. However, these factors are not observable to the traders in the Chinese market. In addition, $\mathrm{Gu}$ and Zhou find that the main stylized facts can be reproduced if one uses a Poisson process for order cancellation (Gu and Zhou 2009a). What is important in the LOB dynamics is the rate of cancellation, together with the rates of limit order placement and market order placement. Hence, we adopt a probability cancellation method in the model, as first reported by Gu et al. (2013), which can capture the proper rate of order cancellation.

The position of an order on the LOB at time $t$ is fully determined by its price level or spatial position $l(t)$ (space dimension) and its temporal position $y(l, t)$ (time dimension) in the order queue at the $l$-th level (Gu et al. 2013, figure 1). First, we define the relative price levels $X_{t}$,

$$
X_{t}=\frac{l(t)}{L_{b, s}(t)},
$$

where $l(t)$ is the price level in which a cancellation occurs in the LOB at event time $t$ and $L_{b, s}(t)$ is the total number of price levels existing in the buy or sell LOB. The relative level $X_{t}$ varies in the range $(0,1]$. We note that the relative price levels $X_{t}$ defined in Eq. (11) is different from the relative price $x_{t}$ defined in Eq. (2) in the order placement process. A small value of $X$ refers to a cancellation happening close to the same best, while a large value of $X$ means that a cancellation occurs far from the same best. Figure 5 a presents the PDF $f(X)$ for both canceled buy and sell orders of stock 000001 . As shown by Gu et al. (2013), the PDF $f(X)$ of relative price levels follows a rescaled log-normal distribution 

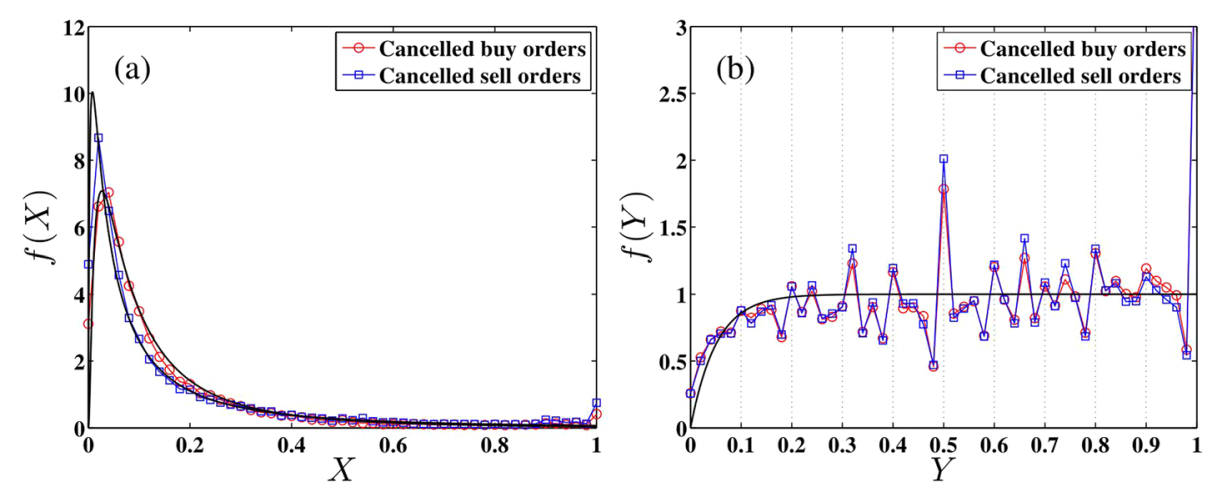

Fig. 5 Determination of the order cancellation process. a Probability density functions $f(X)$ of relative price levels on the LOB for both canceled buy and sell orders of stock 000001 . The solid lines are fits to the rescaled log-normally distribution. $\mathbf{b}$ Probability density functions $f(Y)$ of relative temporal positions at all price levels for both canceled buy and sell orders of the same stock. The solid line is the fit to an exponential function

Table 1 Hurst exponents $H_{s}$ of order directions for the 32 A-share stocks and 11 B-share stocks estimated using the detrending moving average (DMA) method

\begin{tabular}{llllllll}
\hline Stock & $\boldsymbol{H}_{s, \boldsymbol{A}}$ & Stock & $\boldsymbol{H}_{s, \boldsymbol{B}}$ & Stock & $\boldsymbol{H}_{s, \boldsymbol{A}}$ & Stock & $\boldsymbol{H}_{s, \boldsymbol{A}}$ \\
\hline 000002 & $0.904 \pm 0.005$ & 200002 & $0.844 \pm 0.001$ & 000001 & $0.895 \pm 0.008$ & 000778 & $0.842 \pm 0.005$ \\
000012 & $0.834 \pm 0.007$ & 200012 & $0.812 \pm 0.003$ & 000009 & $0.866 \pm 0.010$ & 000800 & $0.871 \pm 0.005$ \\
000016 & $0.841 \pm 0.005$ & 200016 & $0.759 \pm 0.005$ & 000021 & $0.824 \pm 0.010$ & 000825 & $0.906 \pm 0.003$ \\
000024 & $0.832 \pm 0.005$ & 200024 & $0.751 \pm 0.009$ & 000027 & $0.891 \pm 0.006$ & 000839 & $0.859 \pm 0.009$ \\
000429 & $0.827 \pm 0.003$ & 200429 & $0.816 \pm 0.004$ & 000063 & $0.856 \pm 0.008$ & 000858 & $0.885 \pm 0.003$ \\
000488 & $0.814 \pm 0.004$ & 200488 & $0.814 \pm 0.009$ & 000066 & $0.829 \pm 0.009$ & 000898 & $0.884 \pm 0.007$ \\
000539 & $0.852 \pm 0.004$ & 200539 & $0.870 \pm 0.002$ & 000088 & $0.844 \pm 0.005$ & 000917 & $0.815 \pm 0.006$ \\
000541 & $0.862 \pm 0.002$ & 200541 & $0.774 \pm 0.003$ & 000089 & $0.864 \pm 0.003$ & 000932 & $0.876 \pm 0.005$ \\
000550 & $0.834 \pm 0.007$ & 200550 & $0.781 \pm 0.009$ & 000406 & $0.830 \pm 0.008$ & 000956 & $0.848 \pm 0.007$ \\
000581 & $0.892 \pm 0.002$ & 200581 & $0.799 \pm 0.006$ & 000709 & $0.860 \pm 0.006$ & 000983 & $0.853 \pm 0.004$ \\
000625 & $0.806 \pm 0.009$ & 200625 & $0.845 \pm 0.005$ & 000720 & $0.819 \pm 0.002$ & & \\
\hline
\end{tabular}

The mean Hurst exponent is $\left\langle H_{s}\right\rangle=0.841 \pm 0.036$ for all stocks, $\left\langle H_{s, A}\right\rangle=0.854 \pm 0.027$ for A shares, and $\left\langle H_{s, B}\right\rangle=0.806 \pm 0.036$ for B-shares

$$
f(X)=\frac{1}{z} \frac{1}{\sqrt{2 \pi} \sigma X} \exp \left[-\frac{(\ln X-\mu)^{2}}{2 \sigma^{2}}\right]
$$

where $\mu$ is the location parameter, $\sigma$ is the scaling parameter, and $z$ is the normalization constant ensuring that $\int_{0}^{1} f(X) d X=1$. Using the least-squares fitting method, we obtain $\mu=-2.36$ and $\sigma=1.13$ for canceled buy orders and $\mu=-2.49$ and $\sigma=1.52$ for canceled sell orders.

After the price level, $l$ of the cancelation is determined, we need to determine which order will be canceled at the price level. Denote $y(l, t)$ as the temporal position of a canceled order in the queue of the $l$-th price level at time $t$. An order with $y(l, t)=1$ is the order placed the earliest in the queue. To remove the number impact of orders stored at the $l$-th price level, we analyze the relative temporal position $Y(l, t)$ instead of $y(l, t)$, 


$$
Y(l, t)=\frac{y(l, t)}{N_{b, s}(l, t)},
$$

where $Y(l, t)$ varies in the range $(0,1]$ and $N_{b, s}(l, t)$ is the total number of orders stored at the $l$-th price level on the buy or sell LOB at time $t$. Since the PDF $f(Y)$ at each price level in the LOB has a similar shape, we aggregate the data $Y(x, t)$ at all price levels together and treat them as an ensemble. The ensemble PDFs $f(Y)$ for both canceled buy and sell orders of stock 000001 are presented in Fig. 5b. The function $f(Y)$ is close to zero when the relative temporal position $Y$ approaches zero. When $Y$ increases, the PDF first increases rapidly in the range $Y \leq 0.1$ and then fluctuates around a constant level until the end of the LOB. These observations indicate an interesting feature that patient traders have better self-discipline since they place their orders early at certain levels and are less prone to cancel the orders. The $f(Y)$ value is extremely large when $Y=1$, which indicates that the latest placed orders are more likely to be canceled. We can apply an exponential function to fit the PDF of relative temporal position $Y$ as follows,

$$
f(Y)=\frac{1}{z}\left(1-e^{\gamma Y}\right),
$$

where $\gamma$ is the exponent and $z=\left(\gamma+1-e^{\gamma}\right) / \gamma$ is the normalization factor. Using the least-squares fitting method, we obtain $\gamma=-33.78$ for canceled buy orders and $\gamma=-36.57$ for canceled sell orders.

\section{Model specification and validation}

\section{Model specification}

The placed orders can be regarded as contracts for investors willing to buy or sell certain stock shares at a certain price. Once an order is placed, it will be organized in a queue in the LOB. It is clear that the LOB has two opposite sides, that is, buy LOB and sell LOB. Buy orders in the buy LOB are arranged by decreasing the order price and the highest price of the order at the top is called the best bid. In the sell LOB, sell orders are arranged by increasing the order price and the lowest price at the top is called best ask or best offer. If orders have the same order price, they will be arranged based on the placement time. Older orders have priority.

Order cancellation also plays an important role in the price formation of the stock market. If orders are not fully filled, they are usually canceled from the LOB based on investor decisions. When limit orders at the best price are canceled completely, mid-price and spread will change as well. If cancellation occurs inside the LOB, it also affects the shape of the LOB and has potential effects on price formation.

The prices are formed due to the order placement and cancellation processes. Hence, the order-driven model contains these two independent processes. In the order placement process, three ingredients of an order are considered: order direction, order price, and order size.

In the model, we use the event time instead of actual time for simplification and assume that there are $N(T)$ steps (event times) of order placements on a trading day $T$. The price formation process is carried out as follows: 
We first generate an order direction (sign) series $\left\{s_{i}: i=1,2, \ldots, N(T)\right\}$ containing just two elements " +1 " for buy orders and " -1 " for sell orders with the memory effect characterized by the Hurst exponent $H_{s}$ from real data. There are many different algorithms for the generation of fractional Brownian motions (FBMs) (Bardet et al. 2003). We adopt the wavelet-based algorithm to generate FBMs of size $N(T)+1$ (Abry and Sellan 1996), which is an excellent FBM generator especially for small Hurst exponents (Ni et al. 2009; Shao et al. 2012, 2015; Qian et al. 2015). The sign sequence of the increments of the generated FBM is assigned to $\left\{s_{i}\right\}$.

We then generate a relative price series $\left\{x_{i}: i=1,2, \ldots, N(T)\right\}$ with given degree of long memory quantified by the Hurst index $H_{x}$ (Gu and Zhou 2009a). A sequence of relative prices $\left\{x_{i, 0}: i=1,2, \ldots, N(T)\right\}$ is drawn from the probability distribution of real data presented in Fig. 2, in which $x_{i, 0}$ is obtained by solving $z_{i}=F\left(x_{i, 0}\right)$, where $\left\{z_{i}: i=1,2, \ldots, N(T)\right\}$ is a sequence of random numbers drawn from the uniform distribution defined in $[0,1]$ and $F(x)$ is the cumulative distribution of $f(x)$ (Press et al. 1996). To introduce a memory effect to the relative price series $\left\{x_{0}\right\}$ with the Hurst exponent $H_{x}$, we simulate a FBM with $H_{x}$ and record its increments as $\left\{y_{i}: i=1,2, \ldots, N(T)\right\}$. The sequence $\left\{x_{i, 0}: i=1,2, \ldots, N(T)\right\}$ is rearranged such that $x_{i}$ ranks the $n$-th in sequence $\left\{x_{i}: i=1,2, \ldots, N(T)\right\}$ if and only if $y_{i}$ ranks the $n$-th in the $\left\{y_{i}: i=1,2, \ldots, N(T)\right\}$ sequence (Bogachev et al. 2007; Zhou 2008). A detrending moving average analysis of $x_{i}$ confirms that its DMA scaling exponent is very close to $H_{x}$.

Finally, we generate the order size sequence $\left\{v_{i}: i=1,2, \ldots, N(T)\right\}$ from a normal distribution with the average order size $\left\langle v\left(x_{i}\right)\right\rangle$ and its standard deviation $\beta\left(x_{i}\right) x_{i}$, where, for each $x_{i}$, the corresponding $\left\langle v\left(x_{i}\right)\right\rangle$ and $\beta\left(x_{i}\right)$ are determined according to their relations to the relative price depicted in Fig. 4.

Having generated three ingredients $\left\{s_{i}\right\},\left\{x_{i}\right\}$ and $\left\{v_{i}\right\}$ of an order, we continue performing the price formation process. For an order placed at the $t$-th step (event time) $(t=1,2, \ldots, N(T))$, we transform Eq. (2) for buy orders $\left(s_{i}=+1\right)$ and sell orders $\left(s_{i}=-1\right)$ to obtain the order price $p_{i}$, which is rounded to two decimals since the tick size is 0.01 yuan in the Chinese stock market. The $t$-th order is compared with the current buy or sell LOB to determine whether it is fully or partly executed or stored in the LOB according to the price-time priority mechanism of continuous double auction. If a transaction occurs, the mid-price $m(t)$ defined as the mean value of the best bid and best ask is recorded.

At each step, we check whether a cancellation occurs according to a Poisson process with its characteristic parameter obtained from the real data (the cancellation probability is $19 \%$ for stock 000001). If cancellation occurs, we first determine the price level $X$ of the cancellation location in the LOB based on the log-normal distribution presented in of Fig. 5a and then obtain the cancellation position $Y$ at the price level $X$ according to the exponential distribution illustrated in Fig. 5b.

To avoid the buy or sell LOB becoming too empty, one needs to impose additional regulation that there are at least two orders at each side of the LOB after a transaction or cancellation (Mike and Farmer 2008; Gu and Zhou 2009a).

After simulating $N(T)$ steps of order placements, the price formation process is completed on the $T$-th trading day. We can start a new simulation on the $(T+1)$-th trading day with the same process. We mention that on the $(T+1)$-th trading day the maximum 

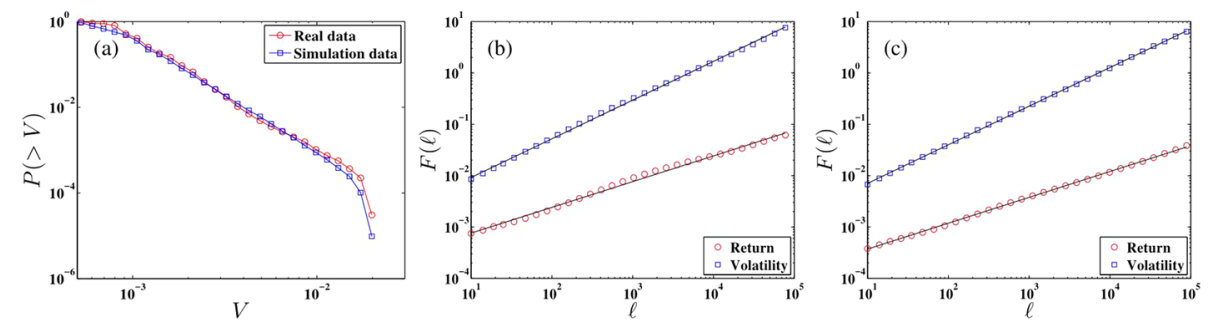

Fig. 6 Model validation. a Empirical cumulative distributions of volatility of simulated data and stock 000001 Both distributions have power-law tails. b DMA fluctuation functions $F(\ell)$ of the return and volatility series for simulation data. c DMA fluctuation functions $F(\ell)$ of the return and volatility series of Stock 000001 . The curves of volatility in $\mathbf{b}, \mathbf{c}$ have been shifted vertically for clarity

allowable price $p_{\max }(T+1)=\left(1+\phi_{+}\right) p_{\mathrm{c}}(T)$ and the minimum allowable price $p_{\min }(T+1)=\left(1+\phi_{-}\right) p_{\mathrm{c}}(T)$, where $p_{\mathrm{c}}(T)$ is the closing price on trading day $T$, which is the average of the last 100 values of the simulated mid-prices $m(j)$. According to the $\pm 10 \%$ price limit rule in the Chinese stock market, we use $\phi_{+}=0.10$ and $\phi_{-}=-0.10$ for model validation.

\section{Model validation}

When a model is built, one needs to calibrate it with known stylized facts (Li et al. 2014). The most universal stylized facts in stock markets are the absence of autocorrelations in the returns, power-law tails in the return distribution and long memory in the volatility time series (Cont 2001). The modified Mike-Farmer model of Gu and Zhou (2009a) can well reproduce these three stylized facts. We illustrate briefly the model validation of these three stylized facts. We note that, although there are general validation approaches in agent-based models (Fagiolo et al. 2019; Krichene et al. 2020), it is sufficient to check the main stylized facts (Mike and Farmer 2008; Gu and Zhou 2009a).

We simulate more than two hundred trading days of price formation and obtain a midprice time series $m(t)$. In the simulations, we set $\phi_{+}=0.10$ and $\phi_{-}=-0.10$ as the real stocks. The returns are calculated as the logarithmic differences of mid-price,

$$
R(t)=\ln [m(t) / m(t-1)]
$$

and the volatility is defined as the absolute value of return,

$$
V_{i}=\left|R_{i}\right|
$$

Figure 6a presents the cumulative volatility distributions of the simulated data from the order-driven model and the real data of stock 000001 . The two distributions overlap with each other and have power-law tails. Using an efficient quantitative method, which is based on the Kolmogorov-Smirnov test and the maximum likelihood estimation (MLE) method (Clauset et al. 2009), we confirm the presence of power-law tails

$$
P(>V) \sim V^{-\alpha}
$$

and obtain the tail exponent $\alpha=2.65 \pm 0.02$ for the model and $\alpha=2.96 \pm 0.03$ for the stock. The results comply with the empirical findings of Chinese stocks (Gu et al. 

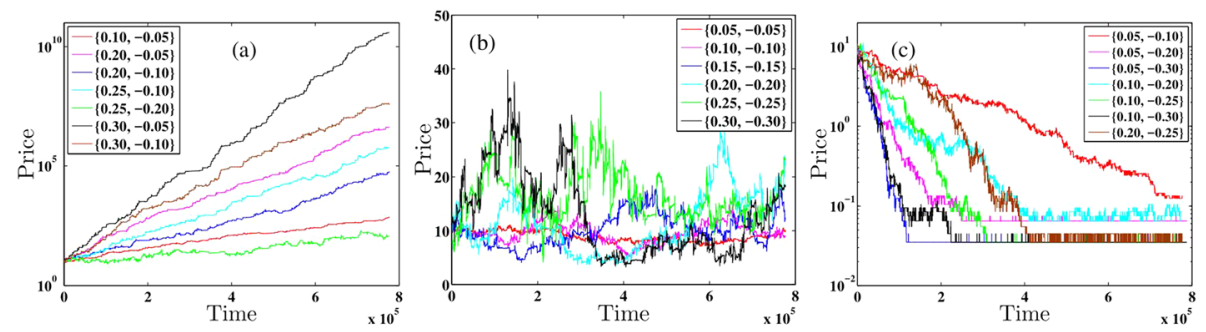

Fig. 7 Price trajectories of the stocks simulated for different combinations of price limits $\left\{\phi_{+}, \phi_{-}\right\}$. a $\left|\phi_{-}\right|<\phi_{+} \cdot \mathbf{b}\left|\phi_{-}\right|=\phi_{+} \cdot \mathbf{c}\left|\phi_{-}\right|>\phi_{+}$

2008). It shows that the tail distribution of volatility obeys the universal inverse cubic law (Gopikrishnan et al. 1998). The discrepancy of tail exponent between the simulated and real stock returns comes from the fact that the method of Clauset et al. (2009) gives $V_{\min }=0.0017$ for the stimulated returns and $V_{\min }=0.0024$ for the real data in which the tail exponents are estimated from the absolute returns that are no less than $V_{\min }$.

We then apply the DMA method to compare the memory effects of simulated data with real data of stock 000001 . Figure $6 \mathrm{~b}$ shows the fluctuation functions $F(\ell)$ with respect to the size scales $\ell$ of simulated return and volatility series. The function $F(\ell)$ scales with the scale $\ell$ as a power law for both curves. Using the least-squares fitting method, we obtain the DMA scaling exponents $H_{R}=0.501 \pm 0.007$ for the return series and $H_{V}=0.753 \pm 0.005$ for the volatility series of the simulation data. In Fig. 6c, we show the fluctuation functions $F(\ell)$ of return and volatility series for the real data and find that $F(\ell)$ also scales with $\ell$ as a power law. We obtain that $H_{R}=0.503 \pm 0.003$ for the return series and $H_{V}=0.752 \pm 0.002$ for the volatility series. The results of simulated returns and volatilities are consistent with stylized facts that return time series is uncorrelated with the Hurst exponent close to 0.5 whereas the volatility series processes long memory with the Hurst exponent significantly greater than 0.5 .

\section{Computational experiments on the effects of asymmetric price limits}

Motivated by the debate on the Draft Guideline on the Trading of Stocks Bearing Risk Warnings, we perform computational experiments to investigate the effects of asymmetric price limit mechanism on the price dynamics and some stylized facts. In the computational experiments, we change the value of $\phi_{+}$from 0.05 to 0.3 with an increment of 0.05 and the value of $\phi_{-}$from -0.3 to -0.05 with the same increment. The initial price is 10 yuan. The tick size is 0.01 yuan.

\section{Vanishing and divergence of price trajectory}

Figure 7 presents the evolution of simulated mid-price for different combinations of $\left\{\phi_{+}, \phi_{-}\right\}:\left|\phi_{-}\right|<\phi_{+}$in panel (a), $\phi_{+}=\left|\phi_{-}\right|$in panel (b), and $\left|\phi_{-}\right|>\phi_{+}$in panel (c). It is evident that the patterns of the price trajectories are similar in each plot but completely different among different plots.

When $\left|\phi_{-}\right|<\phi_{+}$, the price will rise quickly to an unreasonable extent and diverge, as shown in Fig. 7a. The price diverges faster if the up limit is larger or the absolute down 
limit is smaller. Let us take a buy order for example. We can obtain the following equation from Eq. (2),

$$
p_{i}=\left\{\begin{array}{ll}
x\left[p_{\max }(T)-p_{a}(t-1)\right]+p_{a}(t-1) & x \geq 0 \\
x\left[p_{a}(t-1)-p_{\min }(T)\right]+p_{a}(t-1) & x<0
\end{array} .\right.
$$

We know that $p_{i}$ is always proportional to $p_{\max }$ and $p_{\min }$. When an efficient buy market order $(x \geq 0)$ is placed, the price $p_{i}$ increases with the value of $p_{\max }$, which means that the buy price is very aggressive and it tends to push up the stock price when $p_{\max }$ increases. Meanwhile, if a trader places an efficient buy limit order $(x<0), p_{i}$ also increases with the value of $p_{\mathrm{min}}$. It means that the trader places the limit order with a higher price in the limit order book when $p_{\min }$ increases, which makes the stock price grow as well. The explanation for sell orders is similar. Hence, the average return is greater than 0 .

When $\left|\phi_{-}\right|=\phi_{+}$, the price evolves within a reasonable range, as shown in Fig. $7 \mathrm{~b}$. With the increase of $-\phi_{-}$and $\phi_{+}$, the fluctuations of price enhance. We find that there are sharp rises and drops, which are actually bubbles and crashes frequently observed in the Chinese stock market (Zhou and Sornette 2004; Jiang et al. 2010; Wen et al. 2019). This observation is very interesting because it suggests that these artificial traders can also trigger collective behaviors. The model is thus not a zero-intelligence model. Traders' strategies and traits have been captured to a certain extent by the micro regularities used in this model.

When $\left|\phi_{-}\right|>\phi_{+}$, the price drops very quickly, as shown in Fig. 7c. The price decays faster if the up limit is smaller or the absolute down limit is larger. For each curve, there seems to be a lower bound $\mathcal{B}$ such that the price cannot be lower. The presence of this lower bound is actually caused by the presence of down limit and tick size as well as the formation rule of closing prices. Assuming that the closing price on day $T-1$ is $\mathcal{B}$, the minimum price on day $T$ is $\left\lfloor\mathcal{B}\left(1-\left|\phi_{-}\right|\right)\right\rfloor$, where $\lfloor$is the round operator. We note that

$$
\mathcal{B}=\max \left\{\mathcal{B}: 100 \mathcal{B}-\left\lfloor 100 \mathcal{B}\left(1-\left|\phi_{-}\right|\right)\right\rfloor=1\right\} .
$$

In this way, together with the tick size condition

$$
\lfloor 100 \mathcal{B}\rfloor=100 \mathcal{B},
$$

the minimum closing price will be

$$
\left\lfloor 100 \mathcal{B} / 2+\left\lfloor 100 \mathcal{B}\left(1-\left|\phi_{-}\right|\right)\right\rfloor / 2\right\rfloor=\lfloor 100 \mathcal{B}+0.5\rfloor=100 \mathcal{B} .
$$

In order words, the lower bound of the prices is reached at $\mathcal{B}$. It follows from Eq. (19) that

$$
\mathcal{B}=\max \left\{\mathcal{B}: 0.5<100 \mathcal{B}\left|\phi_{-}\right| \leq 1.5\right\},
$$

or

$$
\mathcal{B}=\max \left\{\mathcal{B}: \frac{0.005}{\left|\phi_{-}\right|}<\mathcal{B} \leq \frac{0.015}{\left|\phi_{-}\right|}\right\}
$$


Therefore, for $\phi_{-}=-0.05,-0.10,-0.15,-0.20,-0.25$ and -0.30 , we obtain that $\mathcal{B}=0.30,0.15,0.10,0.07,0.06$ and 0.05 . These values fit the empirical results in Fig. $7 \mathrm{c}$ very well.

Figure 7a shows that, when $\left|\phi_{-}\right|<\phi_{+}$, the price increases exponentially

$$
p_{t} \sim e^{\lambda t}
$$

We introduce the divergence rate $\lambda$ of the price to quantify the impacts of these asymmetric price limit rules. The divergence rates of all cases are estimated as presented in the upper triangle of Table 3 . We propose a linear model for $\lambda\left(\phi_{+}, \phi_{-}\right)$as follows

$$
\lambda\left(\phi_{+}, \phi_{-}\right)=a_{0}+a_{+} \phi_{+}+a_{-}\left|\phi_{-}\right|+\epsilon,
$$

where $\epsilon$ is the noise term. The regression model proves excellent since the adjusted R-square is 0.98 . We obtain that $a_{0}=8.72 \times 10^{-8}$ with a $p$-value of 0.92 , $a_{+}=9.91 \times 10^{-5}$ with a $p$-value of 0.0000 , and $a_{-}=-9.19 \times 10^{-5}$ with a $p$-value of 0.0000 . It indicates that the constant term $a_{0}$ is statistically equal to 0 , and $\phi_{+}$and $\left|\phi_{-}\right|$ have significant impacts on the divergence rate $\lambda$. The divergence rate $\lambda$ increases with $\phi_{+}$and decreases with $\left|\phi_{-}\right|$. In addition, the impact is higher for $\phi_{+}$since $a_{+}>\left|a_{-}\right|$.

To quantify the decay rate of the price for the cases of $\left|\phi_{-}\right|>\phi_{+}$, we calculate the half-lives $t_{1 / 2}$ for all the $\left(\phi_{+}, \phi_{-}\right)$pairs. The half-life is the average of time moments that intersect the horizontal line $p_{t}=p_{1} / 2=5$. The half-lives $t_{1 / 2}$ are digested in the lower triangle of Table 3 . We also propose a linear model for $t_{1 / 2}\left(\phi_{+}, \phi_{-}\right)$as follows

$$
t_{1 / 2}\left(\phi_{+}, \phi_{-}\right)=b_{0}+b_{+} \phi_{+}+b_{-}\left|\phi_{-}\right|+\epsilon,
$$

The regression model proves good since the adjusted R-square is 0.50 . We obtain that $b_{0}=1.22 \times 10^{5}$ with a $p$-value of $0.001, b_{+}=4.56 \times 10^{5}$ with a $p$-value of 0.007 , and $b_{-}=-5.04 \times 10^{5}$ with a $p$-value of 0.004 . It indicates that $\phi_{+}$and $\phi_{-}$have significant impacts on the half-life $t_{1 / 2}$. The half-life $t_{1 / 2}$ also increases with $\phi_{+}$and decreases with $\left|\phi_{-}\right|$. In addition, the impact is higher for $\phi_{-}$since $\left|b_{-}\right|>b_{+}$.

Our computational experiments provide technical evidence against the asymmetric price limit rule (Article VII) proposed in the Draft Guideline on the Trading of Stocks Bearing Risk Warnings. The results show that, with $\phi_{-}=-0.05$ and $\phi_{+}=0.02$, the price of a stock labeled with risk warnings will gradually vanish. These risk-warning stocks will eventually be delisted from the SSE. We note that the results obtained are trivial to some extent because they are mainly caused by the implicit assumption that the relative prices defined in Eq. (2) have the same distribution for symmetric and asymmetric price limit settings. However, these results still have practical importance for policymakers. Certainly, taking policy decisions based on statistical models should be done with care. We note that the results are robust in different experiments. However, the results presented are not averaged over many rounds of experiments because the price trajectories are not comparable in magnitude. 

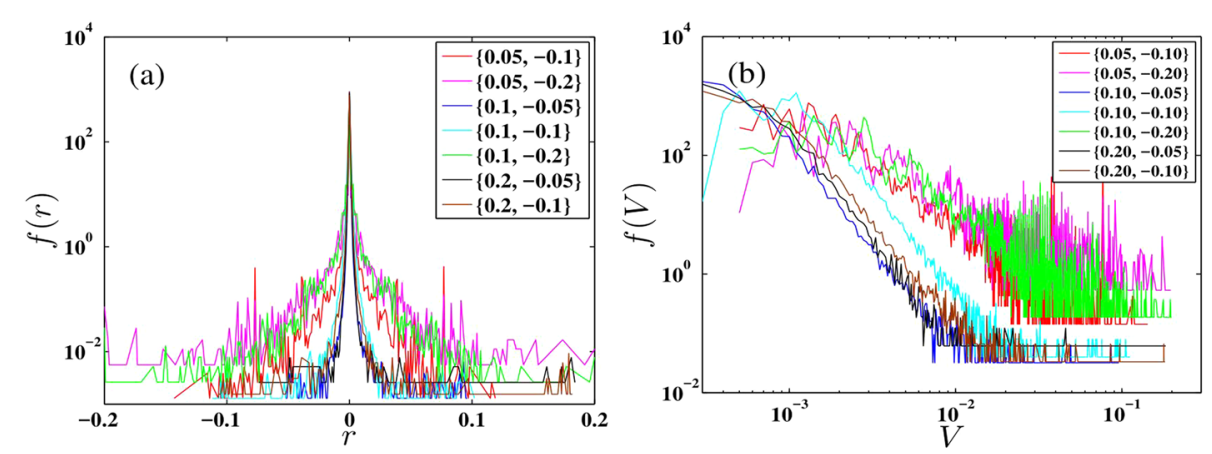

Fig. 8 Empirical distributions of returns in linear-log coordinates (a) and absolute returns in log-log scales (b) for the stocks simulated for different combinations of price limits $\left\{\phi_{+}, \phi_{-}\right\}$

Table 2 Hurst exponents $H_{x}$ of relative prices for the 32 A share stocks and 11 B share stocks estimated using the DMA method

\begin{tabular}{llllllll}
\hline Stock & $\boldsymbol{H}_{\boldsymbol{x}, \boldsymbol{A}}$ & Stock & $\boldsymbol{H}_{\boldsymbol{x}, \boldsymbol{B}}$ & Stock & $\boldsymbol{H}_{\boldsymbol{x}, \boldsymbol{A}}$ & Stock & $\boldsymbol{H}_{\boldsymbol{x}, \boldsymbol{A}}$ \\
\hline 000002 & $0.844 \pm 0.005$ & 200002 & $0.773 \pm 0.002$ & 000001 & $0.847 \pm 0.009$ & 000778 & $0.804 \pm 0.007$ \\
000012 & $0.800 \pm 0.005$ & 200012 & $0.759 \pm 0.004$ & 000009 & $0.814 \pm 0.011$ & 000800 & $0.821 \pm 0.008$ \\
000016 & $0.792 \pm 0.005$ & 200016 & $0.778 \pm 0.003$ & 000021 & $0.814 \pm 0.008$ & 000825 & $0.823 \pm 0.004$ \\
000024 & $0.745 \pm 0.007$ & 200024 & $0.755 \pm 0.002$ & 000027 & $0.802 \pm 0.012$ & 000839 & $0.872 \pm 0.004$ \\
000429 & $0.767 \pm 0.008$ & 200429 & $0.720 \pm 0.005$ & 000063 & $0.840 \pm 0.019$ & 000858 & $0.801 \pm 0.005$ \\
000488 & $0.792 \pm 0.002$ & 200488 & $0.736 \pm 0.005$ & 000066 & $0.808 \pm 0.006$ & 000898 & $0.847 \pm 0.007$ \\
000539 & $0.800 \pm 0.004$ & 200539 & $0.741 \pm 0.008$ & 000088 & $0.776 \pm 0.005$ & 000917 & $0.766 \pm 0.008$ \\
000541 & $0.765 \pm 0.004$ & 200541 & $0.776 \pm 0.002$ & 000089 & $0.756 \pm 0.008$ & 000932 & $0.800 \pm 0.009$ \\
000550 & $0.828 \pm 0.004$ & 200550 & $0.775 \pm 0.004$ & 000406 & $0.787 \pm 0.005$ & 000956 & $0.861 \pm 0.006$ \\
000581 & $0.819 \pm 0.002$ & 200581 & $0.793 \pm 0.002$ & 000709 & $0.807 \pm 0.005$ & 000983 & $0.789 \pm 0.004$ \\
000625 & $0.813 \pm 0.005$ & 200625 & $0.763 \pm 0.006$ & 000720 & $0.841 \pm 0.003$ & & \\
\hline
\end{tabular}

The mean Hurst exponent is $\left\langle H_{x}\right\rangle=0.796 \pm 0.035$ for all stocks, $\left\langle H_{x, A}\right\rangle=0.8075 \pm 0.0301$ for A shares, and $\left\langle H_{x, B}\right\rangle=0.7606 \pm 0.0204$ for $B$ shares

Table 3 Estimated divergence rates of the price trajectories when $\left|\phi_{-}\right|<\phi_{+}$and the half-lives $t_{1 / 2}$ when $\left|\phi_{-}\right|>\phi_{+}$

\begin{tabular}{lllllll}
\hline & $\mathbf{0 . 0 5}$ & $\mathbf{0 . 1 0}$ & $\mathbf{0 . 1 5}$ & $\mathbf{0 . 2 0}$ & $\mathbf{0 . 2 5}$ & $\mathbf{0 . 3 0}$ \\
\hline-0.05 & & 0.520 & 1.050 & 1.630 & 2.041 & 2.508 \\
-0.10 & 10.80 & & 0.543 & 1.063 & 1.619 & 1.977 \\
-0.15 & 4.467 & 6.885 & & 0.593 & 1.117 & 1.648 \\
-0.20 & 2.828 & 4.477 & 1.688 & & 0.562 & 1.000 \\
-0.25 & 2.588 & 4.971 & 5.662 & 9.713 & & 0.854 \\
-0.30 & 1.866 & 1.965 & 2.871 & 3.366 & 7.983 & \\
\hline
\end{tabular}

The first row shows the $\phi_{+}$values, whereas the first column shows the $\phi_{-}$values. The upper triangle presents the convergence rates $\lambda$ (multiplied by $10^{5}$ ), while the lower triangle presents the half-lives $t_{1 / 2}$ (multiplied by $10^{-4}$ )

\section{Return distributions}

With several example combinations of price limits $\left\{\phi_{+}, \phi_{-}\right\}$, Fig. 8a shows the empirical distributions of returns, while Fig. 8b illustrates the empirical distributions of absolute returns, which exhibit evident power-law tails. We unveil two clusters of 
Table 4 Estimated tail exponents of the simulated returns for different price limit combinations

\begin{tabular}{lllllll}
\hline & $\mathbf{0 . 0 5}$ & $\mathbf{0 . 1 0}$ & $\mathbf{0 . 1 5}$ & $\mathbf{0 . 2 0}$ & $\mathbf{0 . 2 5}$ & $\mathbf{0 . 3 0}$ \\
\hline-0.05 & 3.32 & 3.36 & 3.37 & 3.44 & 3.48 & 3.53 \\
-0.10 & 1.94 & 3.41 & 3.51 & 3.43 & 3.52 & 3.50 \\
-0.15 & 1.92 & 2.02 & 3.34 & 3.53 & 3.54 & 3.53 \\
-0.20 & 1.96 & 1.99 & 2.02 & 3.41 & 3.54 & 3.54 \\
-0.25 & 1.91 & 2.08 & 2.03 & 2.12 & 3.20 & 3.45 \\
-0.30 & 2.01 & 2.02 & 2.11 & 2.06 & 2.11 & 2.72 \\
\hline
\end{tabular}

The first row shows the $\phi_{+}$values, whereas the first column shows the $\phi_{-}$values

Table 5 Average returns for different price limit combinations

\begin{tabular}{rrrrrrr}
\hline & $\mathbf{0 . 0 5}$ & $\mathbf{0 . 1 0}$ & $\mathbf{0 . 1 5}$ & $\mathbf{0 . 2 0}$ & $\mathbf{0 . 2 5}$ & \multicolumn{1}{c}{$\mathbf{0 . 3 0}$} \\
\hline-0.05 & 0.017 & 0.544 & 1.098 & 1.721 & 2.188 & 2.550 \\
-0.10 & -0.558 & 0.008 & 0.551 & 1.087 & 1.666 & 1.950 \\
-0.15 & -1.388 & -0.746 & -0.022 & 0.582 & 1.144 & 1.674 \\
-0.20 & -2.181 & -1.384 & -0.719 & -0.052 & 0.462 & 0.934 \\
-0.25 & -2.990 & -2.158 & -1.433 & -0.843 & -0.190 & 0.494 \\
-0.30 & -4.003 & -3.593 & -2.563 & -2.121 & -1.056 & -0.324 \\
\hline
\end{tabular}

The first row shows the $\phi_{+}$values, whereas the first column shows the $\phi_{-}$values. The values of the average returns have been multiplied by $10^{5}$ for better presentation

distributions. The distributions for $\left|\phi_{-}\right| \leq \phi_{+}$(first group) are relatively narrow in the bulks and their tail exponents have comparable values. The distributions for $\left|\phi_{-}\right|>\phi_{+}$ (second group) are relatively broad in the bulks and their tail exponents are also comparable but different from the first group.

The presence of power-law tails observed in Fig. 8b is confirmed by the method of Clauset et al. (2009). The estimated tail exponents for all the price limit combinations under investigation are depicted in Table 4. Consistent with Fig. 8, the tail exponents can be divided into two groups, in which $\langle\alpha\rangle=2.02 \pm 0.07$ for $\left|\phi_{-}\right|>\phi_{+}$and $\langle\alpha\rangle=3.41 \pm 0.18$ for $\left|\phi_{-}\right| \leq \phi_{+}$. To further understand the impacts of $\alpha\left(\phi_{+}, \phi_{-}\right)$, we regress the following linear equation:

$$
\alpha\left(\phi_{+}, \phi_{-}\right)=c_{0}+c_{+} \phi_{+}+c_{-}\left|\phi_{-}\right|+\epsilon .
$$

For all the combinations of price limits $\left\{\phi_{+}, \phi_{-}\right\}$, we obtain that $c_{0}=2.85, c_{+}=4.88$, and $c_{-}=-4.98$, whose $p$-values are all less than 0.0000 . The adjusted R-square is 0.70 and the MSE is 0.1503. Although all the coefficients are significantly different from 0 and the goodness-of-fit is high, it is equivalently fitting "two points" due to the clear separation of the tail exponents and the closeness of the tail exponents in each group. For the combinations with $\left|\phi_{-}\right|>\phi_{+}$, we obtain that $c_{0}=1.87$ with a $p$-value of 0.0000 , $c_{+}=0.75$ with a $p$-value of 0.0015 , and $c_{-}=0.27$ with a $p$-value of 0.1748 . The adjusted $\mathrm{R}$-square is 0.69 and the MSE is 0.0015 . For the combinations with $\left|\phi_{-}\right| \leq \phi_{+}$, we obtain that $c_{0}=3.34$ with a $p$-value of $0.0000, c_{+}=0.53$ with a $p$-value of 0.0429 , and $c_{-}=0.18$ with a $p$-value of 0.4491 . The adjusted R-square is 0.36 and the MSE is 0.0024 . The results suggest that an increase of $\left|\phi_{-}\right|$or $\phi_{+}$will increase the tail exponent $\alpha$, expect for $\phi_{-}$in the $\left|\phi_{-}\right| \leq \phi_{+}$cases. This finding is reasonable since wider price limits will lead to more returns with larger magnitudes. 
Table 6 Estimated Hurst exponents of the simulated returns for different price limit combinations

\begin{tabular}{lllllll}
\hline & $\mathbf{0 . 0 5}$ & $\mathbf{0 . 1 0}$ & $\mathbf{0 . 1 5}$ & $\mathbf{0 . 2 0}$ & $\mathbf{0 . 2 5}$ & $\mathbf{0 . 3 0}$ \\
\hline-0.05 & $0.45 \pm 0.01$ & $0.50 \pm 0.01$ & $0.53 \pm 0.01$ & $0.55 \pm 0.02$ & $0.56 \pm 0.01$ & $0.57 \pm 0.01$ \\
-0.10 & $0.43 \pm 0.01$ & $0.50 \pm 0.02$ & $0.53 \pm 0.01$ & $0.55 \pm 0.01$ & $0.56 \pm 0.01$ & $0.56 \pm 0.02$ \\
-0.15 & $0.45 \pm 0.01$ & $0.45 \pm 0.01$ & $0.52 \pm 0.02$ & $0.55 \pm 0.01$ & $0.56 \pm 0.02$ & $0.57 \pm 0.01$ \\
-0.20 & $0.46 \pm 0.01$ & $0.46 \pm 0.01$ & $0.47 \pm 0.01$ & $0.54 \pm 0.02$ & $0.55 \pm 0.02$ & $0.56 \pm 0.01$ \\
-0.25 & $0.48 \pm 0.02$ & $0.48 \pm 0.01$ & $0.48 \pm 0.01$ & $0.48 \pm 0.01$ & $0.54 \pm 0.02$ & $0.56 \pm 0.02$ \\
-0.30 & $0.49 \pm 0.01$ & $0.50 \pm 0.02$ & $0.49 \pm 0.01$ & $0.50 \pm 0.01$ & $0.49 \pm 0.01$ & $0.52 \pm 0.03$ \\
\hline
\end{tabular}

The first row shows the $\phi_{+}$values, whereas the first column shows the $\phi_{-}$values

In Table 5, we present the average returns for different price limit combinations. When $\left|\phi_{-}\right|<\phi_{+}$, the average returns are positive. When $\left|\phi_{-}\right|>\phi_{+}$, the average returns are negative. When $\left|\phi_{-}\right|=\phi_{+}$, the average return decreases monotonically from positive to negative with the increase of $\phi_{+}$. We use the following linear model to fit the data

$$
\langle r\rangle=d_{0}+d_{+} \phi_{+}+d_{-}\left|\phi_{-}\right|+\epsilon
$$

and obtain that $d_{0}=0.0041 \times 10^{-4}$ with a $p$-value of $0.7363, d_{+}=1.2380 \times 10^{-4}$ with a $p$-value of 0.0000 , and $d_{-}=-1.4147 \times 10^{-4}$ with a $p$-value of 0.0000 . The adjusted $\mathrm{R}$-square is 0.98 , indicating an excellent linear relationship between the average return and the two price limits.

\section{Hurst exponents of returns}

We perform DMA analysis on the return time series for all the combinations of price limits. The estimated Hurst exponents are presented in Table 6. Some characteristic features can be derived from the table, which can be well captured by the linear model:

$$
H_{r}=e_{0}+e_{+} \phi_{+}+e_{-}\left|\phi_{-}\right|+\epsilon .
$$

For the combinations with $\left|\phi_{-}\right|>\phi_{+}$, we obtain that $e_{0}=0.40$ with a $p$-value of 0.0000 , $e_{+}=0.01$ with a $p$-value of 0.3912 , and $e_{-}=0.30$ with a $p$-value of 0.0000 . The adjusted R-square is 0.97 and the MSE is 0.000010 . Hence, $H_{r}$ does not depend on $\phi_{+}$since its coefficient is insignificant, but depends on $\left|\phi_{-}\right|$. The Hurst exponent of returns increases with $\left|\phi_{-}\right|$. For the combinations with $\left|\phi_{-}\right|<\phi_{+}$, we obtain that $e_{0}=0.49$ with a $p$-value of $0.0000, e_{+}=0.27$ with a $p$-value of 0.0000 , and $e_{-}=-0.03$ with a $p$-value of 0.4598 . The adjusted R-square is 0.80 and the MSE is 0.000066 . It shows that $H_{r}$ does not depend on $\phi_{-}$. Rather, the Hurst exponent of returns increases with $\left|\phi_{+}\right|$. For the combinations with $\left|\phi_{-}\right|=\phi_{+}$, we observe a humped shape for the dependence of $H_{r}$ with respect to $\phi_{+}$. Despite the statistically significant trends, the Hurst indexes are all close to 0.5 . The slight deviations are reasonable because of the non-arbitrage nature of the intraday returns caused by the $T+1$ trading mechanism (Zhou et al. 2017).

\section{Hurst exponents of volatilities}

We also perform DMA analysis on the volatility time series for all the combinations of price limits. The estimated Hurst exponents are presented in Table 7. We also find some intriguing features, which can be well captured by the linear model: 
Table 7 Estimated Hurst exponents of the simulated volatilities for different price limit combinations

\begin{tabular}{lllllll}
\hline & $\mathbf{0 . 0 5}$ & $\mathbf{0 . 1 0}$ & $\mathbf{0 . 1 5}$ & $\mathbf{0 . 2 0}$ & $\mathbf{0 . 2 5}$ & $\mathbf{0 . 3 0}$ \\
\hline-0.05 & $0.71 \pm 0.01$ & $0.78 \pm 0.01$ & $0.77 \pm 0.01$ & $0.75 \pm 0.02$ & $0.75 \pm 0.02$ & $0.77 \pm 0.01$ \\
-0.10 & $0.68 \pm 0.01$ & $0.72 \pm 0.01$ & $0.76 \pm 0.02$ & $0.76 \pm 0.02$ & $0.76 \pm 0.02$ & $0.76 \pm 0.01$ \\
-0.15 & $0.70 \pm 0.01$ & $0.71 \pm 0.02$ & $0.72 \pm 0.02$ & $0.75 \pm 0.01$ & $0.77 \pm 0.03$ & $0.77 \pm 0.01$ \\
-0.20 & $0.70 \pm 0.02$ & $0.71 \pm 0.01$ & $0.73 \pm 0.02$ & $0.75 \pm 0.01$ & $0.75 \pm 0.02$ & $0.76 \pm 0.01$ \\
-0.25 & $0.73 \pm 0.02$ & $0.73 \pm 0.02$ & $0.73 \pm 0.02$ & $0.74 \pm 0.02$ & $0.75 \pm 0.02$ & $0.76 \pm 0.02$ \\
-0.30 & $0.73 \pm 0.02$ & $0.75 \pm 0.02$ & $0.73 \pm 0.03$ & $0.75 \pm 0.02$ & $0.75 \pm 0.02$ & $0.77 \pm 0.02$ \\
\hline
\end{tabular}

The first row shows the $\phi_{+}$values, whereas the first column shows the $\phi_{-}$values

$$
H_{V}=f_{0}+f_{+} \phi_{+}+f_{-}\left|\phi_{-}\right|+\epsilon \text {. }
$$

For the combinations with $\left|\phi_{-}\right| \geq \phi_{+}$, we obtain that $f_{0}=0.68$ with a $p$-value of 0.0000 , $f_{+}=0.16$ with a $p$-value of 0.0001 , and $f_{-}=0.13$ with a $p$-value of 0.0006 . The adjusted R-square is 0.80 and the MSE is 0.000088 . In this case, the Hurst exponent of volatility increases with $\phi_{+}$and $\left|\phi_{-}\right|$and the impact of $\phi_{+}$is larger than $\left|\phi_{-}\right|$. For the combinations with $\left|\phi_{-}\right|<\phi_{+}$, we obtain that $f_{0}=0.77$ with a $p$-value of $0.0000, f_{+}=-0.02$ with a $p$-value of 0.6775 , and $f_{-}=-0.01$ with a $p$-value of 0.8870 . The adjusted R-square is -0.13 and the MSE is 0.000094 . In this case, the Hurst exponent $H_{V}$ is independent of $\phi_{+}$ and $\left|\phi_{-}\right|$and remains constant.

\section{Conclusion}

We have developed a behavioral order-driven model with price limit rules based on the empirical regularities of the order placement and cancellation processes. In the order placement process, order directions are determined by the unveiled long memory, order prices are determined by the long memory in relative prices and the asymmetric distribution of relative prices, and order sizes are determined by the nonlinear dependence on the relative price. In the order cancellation process, we adopted a Poisson process with the arrival rate determined from real data and the canceled orders determined empirically by their temporal and spatial positions. The model is validated because it can successfully reproduce the main stylized facts in real markets.

Computational experiments uncover that asymmetric setting of price limits will cause the stock price to diverge when the up limit $\phi_{+}$is greater than the absolute down limit $\left|\phi_{-}\right|$and to vanish vice versa. When $\phi_{+}>\left|\phi_{-}\right|$, the price diverges exponentially, and the divergence rate increases linearly with $\phi_{+}$and decreases linearly with $\left|\phi_{-}\right|$. When $\phi_{+}<\left|\phi_{-}\right|$, the price will eventually decay to a lower bound determined by $\left|\phi_{-}\right|$and the tick size. According to these results, setting asymmetric price limits will destroy the market.

For asymmetric price limits, the simulated returns have power-law tails. The tail exponents for $\phi_{+}<\left|\phi_{-}\right|$are significantly smaller than those for $\phi_{+} \geq\left|\phi_{-}\right|$. In addition, the tail exponent $\alpha$ increases linearly with $\phi_{+}$and decreases linearly with $\left|\phi_{-}\right|$. Interestingly, the average return increases linearly with $\phi_{+}$and decreases linearly with $\left|\phi_{-}\right|$. The Hurst exponents $H_{r}$ of returns are all close to 0.5 but have slight deviations. For each case of asymmetric price limits, the Hurst exponent depends linearly 
on the price limits. In both cases, $H_{r}$ increases with $\phi_{+}$and $\left|\phi_{-}\right|$. For volatility, the Hurst exponents $H_{V}$ are constant for $\phi_{+}>\left|\phi_{-}\right|$, and $H_{V}$ increases with $\phi_{+}$and $\left|\phi_{-}\right|$ for $\phi_{+}<\left|\phi_{-}\right|$. It is difficult to test the results of asymmetric price limits because most stock markets in the world have symmetric price limits or no price limit. The order flow data of asymmetric markets are rare. Meanwhile, since the up limit and down limit of most markets are symmetric, it also verifies the conclusion of our results to some extent.

Our EBOD model provides a suitable computational experiment platform for academics, market participants, and policymakers (Farmer and Foley 2009). For academics, the model can be used to study microstructure theories such as the price impacts of transactions, influencing factors of macroscopic properties, the mechanisms of the formation of bubbles, and the triggering of crashes through collective herding behaviors, systemic risks, and so on. For market participants, the model can be used to design optimal strategies of order placement, perform option pricing, estimate valueat-risk with higher precision, and predict the recurrence probability of extreme fluctuations. For policymakers, the model can be used to study the effects of the different settings of price limits on the performance of markets, such as price discovery, market volatility, and market liquidity.

Certainly, the model is still open for further improvements following further efforts of model specification and calibration. Our model deals briefly and indirectly with the learning and adaptation behavior of traders and assumes that traders do not adjust their learning and adaptation behavior when the market environment changes dramatically. In reality, if the data-generating process changes, investors should realize this and adjust their behavior. Relatedly, investors do not believe in the stock market's fundamental value (say, the discounted value of future dividend payments). Such a building block typically anchors the price dynamics of artificial stock markets, preventing that stock prices become extremely high or low. This mechanism might at least partially offset our main results. This issue can be considered in future work to further improve the model and increase the reliability of the computational experiments.

\section{Abbreviations}

EBOD: Empirical behavioral order-driven; LOB: Limit order book; DMA: Detrending moving average.

\section{Acknowledgements}

We are grateful to the editor and anonymous referees for their insightful suggestions. We also thank the Southwestern University of Finance and Economics for providing open access to our article.

\section{Author's contributions}

WXZ conceived the research. GFG, XX, HCX, and WXZ designed the analyses. GFG and WC compiled the data. GFG, XX, and WXZ conducted the analyses. All authors discussed the results. GFG and WXZ wrote the paper. All authors read and approved the final manuscript.

Funding

This work was supported by the National Natural Science Foundation of China (Grants Nos. U1811462, 71671066, and 71532009), and the Fundamental Research Funds for the Central Universities.

Availability of data and materials

We signed a confidentiality agreement with SSEC who provided us the data used in this work. Hence the data will not be shared.

\section{Declarations}

Competing interests

The authors declare that they have no competing interests. 


\section{Author details}

${ }^{1}$ School of Business, East China University of Science and Technology, Shanghai, China. ${ }^{2}$ Research Center for Econophysics, East China University of Science and Technology, Shanghai, China. ${ }^{3}$ College of Management and Economics, Tianjin University, Tianjin, China. ${ }^{4}$ China Center for Social Computing and Analytics, Tianjin University, Tianjin, China. ${ }^{5}$ Shenzhen Stock Exchange, Shenzhen, China. ${ }^{6}$ School of Mathematics, East China University of Science and Technology, Shanghai, China.

Received: 25 August 2020 Accepted: 19 August 2021

Published online: 01 November 2021

\section{References}

Abry P, Sellan F (1996) The wavelet-based synthesis for the fractional Brownian motion proposed by F. Sellan and Y. Meyer: remarks and fast implementation. Appl Comp Harmonic Anal 3:377-383

Arthur WB (1994) Inductive reasoning and bounded rationality. Am Econ Rev 84(2):406-411

Bardet JM, Lang G, Oppenheim G, Philippe A, Stoev S, Taqqu MS (2003) Generators of long-range dependence processes: a survey. In: Doukhan P, Oppenheim G, Taqqu M (eds) Theory and applications of long-range dependence. Birkhauser, New York, pp 579-623

Barunik J, Vacha L, Vosvrda M (2009) Smart predictors in the heterogeneous agent model. J Econ Interact Coord 4(2):163 Biais B, Hillion P, Spatt C (1995) An empirical analysis of the limit order book and the order flow in the Paris Bourse. J Financ 50(5):1655-1689

Bogachev MI, Eichner JF, Bunde A (2007) Effect of nonlinear correlations on the statistics of return intervals in multifractal data sets. Phys Rev Lett 99(24):240601

Bornholdt S (2001) Expectation bubbles in a spin model of markets: intermittency from frustration across scales. Int J Mod Phys C 12(5):667-674

Brennan MJ (1986) A theory of price limits in futures markets. J Financ Econ 16(2):213-233

Brock WA, Hommes CH (1997) A rational route to randomness. Econometrica 65(5):1059-1095

Brock WA, Hommes CH (1998) Heterogeneous beliefs and routes to chaos in a simple asset pricing model. J Econ Dyn Control 22(8-9):1235-1274

Carbone A (2009) Detrending moving average algorithm: a brief review. Science and Technology for Humanity (TIC-STH), pp 691-696

Chakraborti A, Toke IM, Patriarca M, Abergel F (2011) Econophysics review: II. Agent-based models. Quant Financ 11(7):1013-1041

Challet D, Zhang YC (1997) Emergence of cooperation and organization in an evolutionary game. Phys A 246(3-4):407-418

Challet D, Marsili M, Zhang YC (2000) Modeling market mechanism with minority game. Phys A 276(1-2):284-315

Challet D, Marsili M, Zhang YC (2001a) Stylized facts of financial markets and market crashes in minority games. Phys A 294(3-4):514-524

Challet D, Marsili M, Zhang YC (2001 b) Minority games and stylized facts. Phys A 299(1-2):228-233

Challet D, Marsili M, Zhang YC (2005) Minority games: interacting agents in financial markets. Oxford University Press, Oxford

Chiarella C, lori G (2002) A simulation analysis of the microstructure of double auction markets. Quant Financ 2(5):346-353

Chiarella C, He XZ, Wang D (2006) A behavioral asset pricing model with a time-varying second moment. Chaos Solitons Fractals 29(3):535-555

Chiarella C, lori G, Perelló J (2009) The impact of heterogeneous trading rules on the limit order book and order flows. J Econ Dyn Control 33(3):525-537

Chiarella C, He XZ, Zhang M (2011) An analysis of the effect of noise in a heterogeneous agent financial market model. J Econ Dyn Control 35(1):148-162

Chiarella C, He XZ, Shi L, Wei LJ (2017) A behavioural model of investor sentiment in limit order markets. Quant Financ 17(1):71-86

Chowdhury D, Stauffer D (1999) A generalized spin model of financial markets. Eur Phys J B 8(3):477-482

Clauset A, Shalizi CR, Newman MEJ (2009) Power-law distributions in empirical data. SIAM Rev 51(4):661-703

Cont R (2001) Empirical properties of asset returns: stylized facts and statistical issues. Quant Financ 1(2):223-236

Cont R, Bouchaud JP (2000) Herd behavior and aggregate fluctuations in financial markets. Macroecon Dyn 4(2):170-196

Degryse H, Jong F, Ravenswaaij MV, Wuyts G (2005) Aggressive orders and the resiliency of a limit order market. Rev Financ 9(2):201-242

Eguíluz VM, Zimmermann MG (2000) Transmission of information and herd behavior: an application to financial markets. Phys Rev Lett 85(26):5659-5662

Ehrenstein G, Westerhoff F (2006) The working of circuit breakers within percolation models for financial markets. Int J Mod Phys C 17(2):299-304

Fagiolo G, Guerini M, Lamperti F, Moneta A, Roventini A (2019) 31. In: Validation of agent-based models in economics and finance. Springer, pp. 763-787

Farmer JD, Foley D (2009) The economy needs agent-based modelling. Nature 460:685-686

Farmer JD, Patelli P, Zovko II (2005) The predictive power of zero intelligence in financial markets. Proc Natl Acad Sci USA 102(6):2254-2259

Föellmer H (1974) Random economies with many interacting agents. J Math Econ 1(1):51-62

Goettler RL, Parlour CA, Rajan U (2009) Informed traders and limit order markets. J Financ Econ 93(1):67-87 
Gopikrishnan P, Meyer M, Amaral LAN, Stanley HE (1998) Inverse cubic law for the distribution of stock price variations. Eur Phys J B 3(2):139-140

Gu GF, Zhou WX (2009a) Emergence of long memory in stock volatility from a modified Mike-Farmer model. EPL 86(4):48002

Gu GF, Zhou WX (2009b) On the probability distribution of stock returns in the Mike-Farmer model. Eur Phys J B 67(4):585-592

Gu GF, Zhou WX (2010) Detrending moving average algorithm for multifractals. Phys Rev E 82(1):011136

Gu GF, Chen W, Zhou WX (2007) Quantifying bid-ask spreads in the Chinese stock market using limit-order book data. Eur Phys J B 57(1):81-87

Gu GF, Chen W, Zhou WX (2008) Empirical distributions of Chinese stock returns at different microscopic timescales. Phys. A 387(2-3):495-502

Gu GF, Xiong X, Ren F, Zhou WX, Zhang W (2013) The position profiles of order cancellations in an emerging stock market. J Stat Mech 2013(4):P04027

He XZ, Li K (2015) Profitability of time series momentum. J Bank Financ 53:140-157

Hsieh PH, Kim YH, Yang JJ (2009) The magnet effect of price limits: a logit approach. J Empir Financ 16(5):830-837

Iori G (1999) Avalanche dynamics and trading friction effects on stock market returns. Int J Mod Phys C 10(6):1149-1162

Jiang ZQ, Zhou WX (2011) Multifractal detrending moving-average cross-correlation analysis. Phys Rev E 84(1):016106

Jiang ZQ, Chen W, Zhou WX (2008) Scaling in the distribution of intertrade durations of Chinese stocks. Phys A 387(23):5818-5825

Jiang ZQ, Zhou WX, Sornette D, Woodard R, Bastiaensen K, Cauwels P (2010) Bubble diagnosis and prediction of the 2005-2007 and 2008-2009 Chinese stock market bubbles. J Econ Behav Organ 74(3):149-162

Jiang ZQ, Xie WJ, Zhou WX, Sornette D (2019) Multifractal analysis of financial markets: a review. Rep Prog Phys 82(12):125901

Kaizoji T (2000) Speculative bubbles and crashes in stock markets: an interacting-agent model of speculative activity. Phys A 287(3-4):493-506

Kim KA, Rhee SG (1997) Price limit performance: evidence from the Tokyo Stock Exchange. J Financ 52(2):885-901

Krichene H, Inoue H, Isogai T, Chakraborty A (2020) A model of the indirect losses from negative shocks in production and finance. PLOS ONE 15(9):e0239293

Li LS, Geng XY (2012) Design of China's stock market trading mechanism: based on the study of asymmetric price limit system. Res Invest Stud (in Chinese) 31(343):82-94

Li YL, Zhang W, Zhang YJ, Zhang XT, Xiong X (2014) Calibration of the agent-based continuous double auction stock market by scaling analysis. Inf Sci 256:46-56

Lillo F, Farmer JD (2004) The long memory of the efficient market. Stud Nonlin Dyn Econometr 3:1-33

Lillo F, Farmer JD, Mantegna R (2003) Master curve for price impact function. Nature 421(6919):129-130

Lim M, Coggins R (2005) The immediate price impact of trades on the Australian Stock Exchange. Quant Financ. 5(4):365-377

Lux T, Marchesi M (1999) Scaling and criticality in a stochastic multi-agent model of a financial market. Nature 397(6719):498-500

Maslov S (2000) Simple model of a limit order-driven market. Phys A 278(3-4):571-578

Meng H, Ren F, Gu GF, Xiong X, Zhang YJ, Zhou WX et al (2012) Effects of long memory in the order submission process on the properties of recurrence intervals of large price fluctuations. EPL 98(3):38003

Mike S, Farmer JD (2008) An empirical behavioral model of liquidity and volatility. J Econ Dyn Control 32(1):200-234

Mu GH, Chen W, Kertesz J, Zhou WX (2009) Preferred numbers and the distributions of trade sizes and trading volumes in the Chinese stock market. Eur Phys J B 68(1):145-152

$\mathrm{Ni}$ XH, Jiang ZQ, Zhou WX (2009) Degree distributions of the visibility graphs mapped from fractional Brownian motions and multifractal random walks. Phys Lett A 373(42):3822-3826

Pham MC, Duong HN, Lajbcygier P (2017) A comparison of the forecasting ability of immediate price impact models. J Forecast 36(8):898-918

Press W, Teukolsky S, Vetterling W, Flannery B (1996) Numerical recipes in FORTRAN: the art of scientific computing. Cambridge University Press, Cambridge

Qian XY, Liu YM, Jiang ZQ, Podobnik B, Zhou WX, Stanley HE (2015) Detrended partial cross-correlation analysis of two nonstationary time series influenced by common external forces. Phys Rev E 91(6):062816

Shao YH, Gu GF, Jiang ZQ, Zhou WX, Sornette D (2012) Comparing the performance of FA, DFA and DMA using different synthetic long-range correlated time series. Sci Rep 2:835

Shao YH, Gu GF, Jiang ZQ, Zhou WX (2015) Effects of polynomial trends on detrending moving average analysis. Fractals 23(3):1550034

Sornette D (2014) Physics and financial economics (1776-2014): puzzles, Ising and agent-based models. Rep Prog Phys 77(6):062001

Stauffer D (1998) Can percolation theory be applied to the stock market? Ann Phys 7(5-6):529-538

Stigler GJ (1964) Public regulation of the securities markets. J Bus 37(2):117-142

Subrahmanyam A (1994) Circuit breakers and market volatility: a theoretical perspective. J Financ 49(1):237-254

Telser LG (1981) Margins and futures contracts. J Fut Mark 1(2):225-253

Tseng J, Lin CH, Lin CT, Wang SC, Li SP (2010) Statistical properties of agent-based models in markets with continuous double auction mechanism. Phys A 389:1699-1707

Wan YL, Xie WJ, Gu GF, Jiang ZQ, Chen W, Xiong X et al (2015) Statistical properties and pre-hit dynamics of price limit hits in the Chinese stock markets. PLoS ONE 10(4):e0120312

Wan YL, Wang GJ, Jiang ZQ, Xie WJ, Zhou WX (2018) The cooling-off effect of price limits in the Chinese stock markets. Phys. A 505:153-163

Wen F, Xu L, Ouyang G, Kou G (2019) Retail investor attention and stock price crash risk: evidence from China. Int Rev Financ Anal 65:101376

Westerhoff F (2003) Speculative markets and the effectiveness of price limits. J Econ Dyn Control 28(3):493-508 
Westerhoff FH (2006) Technical analysis based on price-volume signals and the power of trading breaks. Int J Theor Appl Financ 9(2):227-244

Westerhoff FH (2008) The use of agent-based financial market models to test the effectiveness of regulatory policies. Jahrb Natl Stat 228(2-3):195-227

Westerhoff F, Franke R (2018) Agent-based models for economic policy design: two illustrative examples. In: Chen SH, Kaboudan M, Du YR (eds) The Oxford handbook of computational economics and finance. Oxford University Press, Oxford, pp 520-558

Wong WK, Liu B, Zeng Y (2009) Can price limits help when the price is falling? Evidence from transactions data on the Shanghai Stock Exchange. China Econ Rev 20(1):91-102

Wu T, Wang Y, Li MX (2017) Post-hit dynamics of price limit hits in the Chinese stock markets. Phys A 465:464-471

Xiong X, Ding D, Yang Y, Zhang YJ (2015) Study on market stability and price limit of Chinese stock index futures market: an agent-based modeling perspective. PLOS ONE 10(11):e0141605

Xu HC, Zhang W, Xiong X, Zhou WX (2014a) Wealth share analysis with "fundamentalist" heterogeneous agents. Abstr Appl Anal 2014:328498

Xu HC, Zhang W, Xiong X, Zhou WX (2014b) An agent-based computational model for China's stock market and stock index futures market. Math Probl Eng 2014:563912

Xu HC, Jiang ZQ, Zhou WX (2017) Immediate price impact of a stock and its warrant: Power-law or logarithmic model? Int J Mod Phys B 31:1750048

Yeh $\mathrm{CH}$, Yang CY (2010) Examining the effectiveness of price limits in an artificial stock market. J Econ Dyn Control 34(10):2089-2108

Yeh CH, Yang CY (2013) Do price limits hurt the market? J Econ Interact Coord 8(1):125-153

Zha Q, Kou G, Zhang H, Liang H, Chen X, Li C et al (2020) Opinion dynamics in finance and business: a literature review and research opportunities. Financ Innov 6(1):44

Zhang XT, Ping J, Zhu T, Li YL, Xiong X (2016) Are price limits effective? An examination of an artificial stock market. PLoS ONE 11(8):e0160406

Zhou WX, Sornette D (2004) Antibubble and prediction of China's stock market and real-estate. Phys A 337(1-2):243-268

Zhou WX (2008) Multifractal detrended cross-correlation analysis for two nonstationary signals. Phys Rev E 77(6):066211

Zhou WX (2012a) Determinants of immediate price impacts at the trade level in an emerging order-driven market. New J Phys 14:023055

Zhou WX (2012b) Universal price impact functions of individual trades in an order-driven market. Quant Financ. 12(8):1253-1263

Zhou WX, Sornette D (2007) Self-organizing Ising model of financial markets. Eur Phys J B 55(2):175-181

Zhou J, Gu GF, Jiang ZQ, Xiong X, Chen W, Zhang W et al (2017) Computational experiments successfully predict the emergence of autocorrelations in ultra-high-frequency stock returns. Comput Econ 50(4):579-594

\section{Publisher's Note}

Springer Nature remains neutral with regard to jurisdictional claims in published maps and institutional affiliations.

\section{Submit your manuscript to a SpringerOpen ${ }^{\circ}$ journal and benefit from:}

- Convenient online submission

- Rigorous peer review

- Open access: articles freely available online

High visibility within the field

Retaining the copyright to your article

Submit your next manuscript at $\boldsymbol{\nabla}$ springeropen.com 